\section{Educar en conductas sexuales saludables: una innovación docente en promoción de la salud}

\author{
MARÍA A. GARCÍA G. ${ }^{1, a}$, GUILLERMO A. CAÑADAS-DE LA FUENTE², \\ EMILIO GONZÁLEZ-JIMÉNEZ², RAFAEL FERNÁNDEZ CASTILLO ${ }^{3}$, \\ INMACULADA GARCÍA-GARCÍA
}

\section{Education about healthy sexual behaviors among high school students}

Background: The need to educate adolescents about healthy sexual behavior motivated the development of an education program on sexuality. Aim: To describe the knowledge of students about sexuality and the implementation of a sexual education project. Material and Methods: Sixty students aged 14 years (34 females), attending the third year of secondary education participated in dynamic workshops about healthy sexual behaviors. Results: The most common reactions of students when facing situations related to sexual behavior were shame, anxiety, fear and lack of knowledge. Group dynamics improved the practical knowledge of students about sexual behavior, allowing them to make informed decisions. Conclusions: These innovative educational techniques should be expanded to other students.

(Rev Med Chile 2011; 139: 1269-1275).

Key words: Adolescent; Sex education; Sexual behavior.

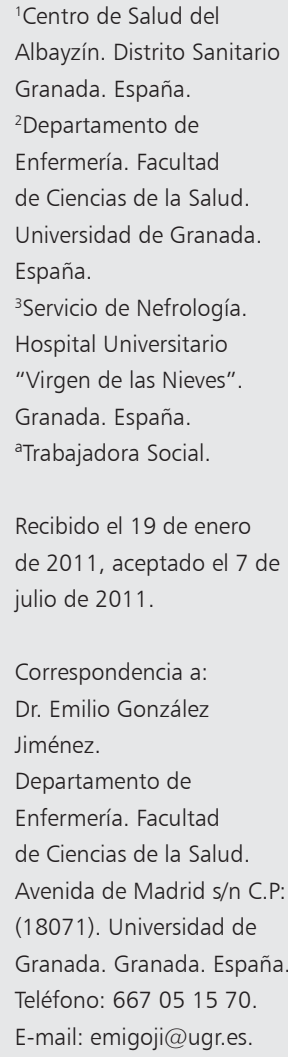

$\mathrm{E}$ l comportamiento sexual en la edad adulta se debe caracterizar por los principios éticos y universales de tolerancia, respeto, ausencia de imposiciones y de discriminaciones, así como disposición empática hacia los demás. En la población adolescente estos principios deben cumplirse aun más, puesto que carecen de los conocimientos y de la experiencia necesarios para afrontar situaciones no deseables ${ }^{1,2}$.

En estas edades existe el riesgo de dejar que otros u otras tomen decisiones respecto a las responsabilidades inherentes a la sexualidad, o bien elegir de forma equivocada por condicionantes diversos, tales como la falta de información, la presión del entorno social, o bien simplemente una mala planificación del futuro en ese momento ${ }^{1}$. Este tipo de circunstancias pueden desembocar en situaciones tales, entre otras, como embarazos no deseados e infecciones de transmisión sexual (ITS) $)^{3,4}$.
Estudios recientes muestran la existencia de ligeras discrepancias de las autoridades sanitarias españolas con respecto a los contenidos que sobre educación sexual se imparten en la enseñanza secundaria obligatoria (ESO). Los manuales exponen información incompleta y contradictoria con respecto a publicaciones científicas recogida en la bibliografía, además de la carencia de precisión en la información a nivel general y en la prevención de ITS en particular. Esta información no ayuda a formar al estudiante y entre otras razones contribuye a que los adolescentes inicien precozmente su actividad sexual basadas en creencias erróneas ${ }^{5}$.

La educación para la sexualidad, en el proceso de atención de enfermería dentro del ámbito educativo, constituye una parte importante de la cartera de servicios de las enfermeras ${ }^{6}$. Además, la educación para la sexualidad realizada por profesionales de enfermería constituye un proceso educativo dirigido a potenciar, promover y educar 
aquellos factores de la sexualidad que inciden directamente sobre la población juvenil en general ${ }^{7,8}$.

Dicha población inicia su actividad sexual en la adolescencia ${ }^{9}$ con los riesgos inherentes a esta etapa de la vida. Además el abuso de alcohol y drogas constituyen algunos de los malos hábitos presentes en esta población ${ }^{10} \mathrm{y}$ forman parte de los principales factores de riesgo que influyen notablemente en la sexualidad, tal y como demuestran estudios recientes ${ }^{11}$.

A partir de una formación en sexualidad y desde un punto de vista holístico o modelo integral $^{12,13}$, el adolescente podría disponer de los recursos necesarios para afrontar, con suficiente madurez, y con minimización de riesgos, los diferentes problemas que deberá solventar en un futuro en situaciones concretas. Dicho futuro estará lleno de incertidumbres, ya que el hecho de estar más preparado no significa que deje de estar expuesto, si bien es cierto que la capacidad de resolución de los problemas, ya mencionados, aumentará de una forma exponencial y permitirá al adolescente ser más consecuente con las decisiones que adopte ${ }^{1}$.

Por tanto, una actitud correctamente aprendida y aplicada acabará por promover conductas saludables, al tiempo que afianza una prevención primaria de salud ${ }^{14}$, considerando las diferencias de género ${ }^{11,15} \mathrm{y}$ socioculturales según cada región de origen ${ }^{16,17}$. Es por ello que está justificado el hecho de impartir y evaluar ${ }^{9}$ una educación para la salud entre la población más joven para implementar conductas de lo que podría ser una sexualidad más saludable ${ }^{18-20}$. Afrontar la sexualidad les preocupa a los adolescentes, aunque por lo general no lo manifiesten abiertamente. El entorno más adecuado para iniciar el proceso de formación sería en los propios centros educativos, puesto que todos los y las adolescentes realizan la ESO y por ello nuestra información incide selectivamente en ese momento del desarrollo humano ${ }^{21}$.

Este trabajo consta de dos partes o etapas: la primera corresponde a un proyecto de educación sexual impartido durante los meses de enero y febrero de 2009 en un Instituto de Enseñanza Secundaria (IES), durante el curso académico 2008-2009. Dicho proyecto se realiza mediante dinámicas de grupo, metodología de uso habitual desde hace tiempo ${ }^{22}$ y adecuada para lograr el objetivo de este trabajo, tal y como exponen otros estudios $^{23,24}$. La segunda, consta de la elaboración y puesta en marcha del proyecto conocido con el nombre de "Forma Joven". Éste consta de varias actividades dedicadas a educación para la salud y el desarrollo de una aplicación vía internet para la resolución de consultas "on line". Este proyecto se implementa durante el curso académico 20092010. El marco legal de este trabajo tiene como referencia la Ley Orgánica de Educación española $(\mathrm{LOE})^{25}$.

\section{Objetivos}

Los objetivos de este estudio fueron los siguientes:

- Describir los conocimientos sobre la sexualidad en los alumnos de $3^{\text {er }}$ curso de un instituto de enseñanza secundaria obligatoria. Implementar un proyecto de educación sexual en adolescentes.

- Realizar un proyecto de educación para la salud incluido en el programa denominado "Forma Joven".

\section{Metodología}

Se realizó un estudio descriptivo, intervencionista y de abordaje cualitativo con preguntas abiertas y dinámicas grupales.

El centro educativo solicitó al centro de salud la actividad formativa en conductas sexuales saludables. Esta actividad fue incluida en el plan docente por el claustro del centro, entre cuyos miembros se encuentra la Asociación de Padres de Alumnos (APA). La comunidad educativa designa el tercer curso de la educación secundaria, cuya edad en su mayoría es de 14 años, edad pertinente para realizar este tipo de actividades.

Para programar la actividad se designa un grupo coordinador, integrado por una enfermera y la trabajadora social, ambas profesionales del Centro de Salud; y por dos profesores del Centro Educativo.

Se implementó un proyecto de educación sexual en el primer semestre del año 2009 y se programó otro proyecto dentro de la propuesta de la Junta de Andalucía (gobierno de la Comunidad Autónoma de Andalucía, España) del programa denominado "Forma Joven" para el curso 20092010.

Las actividades del proyecto de educación sexual se desarrollaron a través de talleres con la 
participación de todo el alumnado. En estos talleres se realizaron técnicas de dinámica de grupo (tormenta de ideas, representación de roles, juegos de mitos, datos...), que tenían como fin educar en materia de salud sexual. Estos talleres se efectuaron durante los meses de enero y febrero de 2009. En ellos se realizaron un total de seis sesiones de 45 minutos de duración en horario lectivo. El taller fue impartido por una enfermera y la trabajadora social del Centro de Salud de la Zona Básica de Salud, coordinadas con dos profesores de la institución docente en el IES Albayzín (barrio histórico de la ciudad de Granada). El horario para desarrollar esta actividad fue el horario de tutoría.

Durante la reunión con los profesores se abordó y trató el cronograma y diseño del estudio a realizar. La información empleada partió de un documento explicativo, tal y como se puede observar en la Tabla 1 , donde se refleja la finalidad del taller y la metodología a emplear con el alumnado, así como el contenido del mismo.

\section{Material y Método}

La población diana estaba integrada por dos grupos de $3^{\circ}$ de ESO del IES con un total de 60 alumnos (34 alumnas y 26 alumnos), que tenían una edad media de 14,1 años. Las sesiones fueron de carácter participativo y dicho alumnado tuvo la oportunidad de mostrar sus intereses al comienzo de las sesiones, a través de un cuestionario con preguntas de tipo abiertas ${ }^{26}$. Dichas preguntas eran las siguientes: - ¿Qué entiendes por sexualidad? - ¿Qué cuestiones de esta materia te gustaría conocer más? -. Los alumnos dispusieron de un tiempo limitado para contestar a estas preguntas. Se realizó un análisis de contenido de las respuestas.

En relación a la puesta en marcha del proyecto "Forma Joven" se mantuvo una reunión con los profesores del grupo coordinador para acordar el contenido de dicho proyecto. En dicha reunión se planificó el contenido de las sesiones que integrarían los talleres. El fin de estas sesiones era el de complementar a los ya iniciados en el curso anterior $^{26}$ (que se describen en este proyecto), y la aplicación para consulta telemática "on line” de estos temas, a través de la página web del centro educativo.

Para la realización de este estudio se cumplieron todas las normas éticas exigidas internacionalmente. Además, previo al inicio del estudio fue necesario contar con la aprobación por parte del Comité Ético de la Delegación Provincial de Educación de Granada.

Tabla 1. Contenidos de los talleres

\begin{tabular}{|c|c|c|c|}
\hline Título de la sesión & Objetivos & Técnicas & Instrumentos \\
\hline Concepto de sexualidad & $\begin{array}{l}\text { Educación sexual y autono- } \\
\text { mía personal }\end{array}$ & $\begin{array}{l}\text { Trabajo individual } \\
\text { Trabajo grupal } \\
\text { Puesta en común }\end{array}$ & $\begin{array}{l}\text { Cuestionario sobre sexua- } \\
\text { lidad }\end{array}$ \\
\hline $\begin{array}{l}\text { Percepciones en torno a la } \\
\text { sexualidad }\end{array}$ & $\begin{array}{l}\text { Reforzar la información ade- } \\
\text { cuada sobre sexualidad }\end{array}$ & $\begin{array}{l}\text { Juego de mitos y datos, } \\
\text { divididos los alumnos en } \\
\text { dos grupos con un portavoz } \\
\text { cada uno }\end{array}$ & $\begin{array}{l}\text { Cuestionario amplio sobre } \\
\text { sexualidad }\end{array}$ \\
\hline Desarrollo sexual & $\begin{array}{l}\text { Comprobación de conoci- } \\
\text { mientos opiniones e intere- } \\
\text { ses sobre desarrollo sexual }\end{array}$ & $\begin{array}{l}\text { Trabajo individual } \\
\text { Trabajo grupal } \\
\text { Puesta en común }\end{array}$ & $\begin{array}{l}\text { Cuestionario sobre sexuali- } \\
\text { dad en la adolescencia }\end{array}$ \\
\hline $\begin{array}{l}\text { Vivencia de los cambios } \\
\text { corporales }\end{array}$ & $\begin{array}{l}\text { Reconocer los principales } \\
\text { cambios psicológicos }\end{array}$ & Analizar texto en grupo & $\begin{array}{l}\text { Cumplimentar cuestionario } \\
\text { sobre el texto }\end{array}$ \\
\hline $\begin{array}{l}\text { Implicaciones psicológicos } \\
\text { de los cambios en ambos } \\
\text { sexos }\end{array}$ & $\begin{array}{l}\text { Empatizar con el sexo } \\
\text { opuesto }\end{array}$ & $\begin{array}{l}\text { Representar los cambios } \\
\text { físico y psicológicos en el } \\
\text { sexo opuesto }\end{array}$ & Representación \\
\hline Fecundidad y sexualidad & $\begin{array}{l}\text { Adquirir conocimientos y } \\
\text { actitudes en relación a la } \\
\text { fecundidad }\end{array}$ & $\begin{array}{l}\text { Juegos de roles sobre el } \\
\text { afrontamiento del embarazo }\end{array}$ & $\begin{array}{l}\text { Cuestionario sobre fecun- } \\
\text { didad }\end{array}$ \\
\hline
\end{tabular}




\section{Resultados}

Se realizaron un total de seis sesiones de 45 minutos aproximadamente cada una de ellas, en las que participaron 60 alumnos de $3^{\circ}$ de ESO (alumnos de 14 años).

La primera encuesta con preguntas abiertas nos orientó en relación con las necesidades individualizadas en función del género. Se detectó que los varones asociaban las relaciones sexuales al acto sexual en sí mismo, mientras que las mujeres abarcaban en las mismas una concepción algo más amplia, en relación con aspectos tales como los procesos fisiológicos inherentes a la sexualidad.

En la parte práctica del taller, la metodología empleada permitió la participación de todo el grupo, con lo que ningún alumno o alumna se marginó. Con el juego de roles, a partir de la falta de conocimientos detectada al inicio del estudio, los participantes pudieron experimentar por un tiempo limitado y en primera persona, situaciones no vividas, así como escenificar la manera de afrontarlas.

Durante las sesiones se expusieron las diferentes formas en que más habitualmente se presentan las conductas sexuales. Las manifestaciones más frecuentes expresadas por el alumnado fueron, en grado leve o moderado, las de vergüenza, ansiedad, miedo y desconocimiento, en cuanto a afrontar determinadas situaciones.

Como continuidad del proyecto de educación sexual se concretó una intervención más amplia al amparo de la propuesta del gobierno de la Comunidad Autónoma, que a través de la Consejería de Educación, la Consejería de Salud y la Consejería para la Igualdad y el Bienestar Social han diseñado para los Institutos de Enseñanza Secundaria una intervención más amplia. Esta intervención se concreta en el proyecto "Forma Joven".

Con los profesores integrantes del grupo coordinador se acordó el contenido del proyecto "Forma Joven". Dicho proyecto consta de las siguientes actividades:

1. Los talleres elegidos estuvieron dedicados a los siguientes temas: alimentación, sexualidad $\mathrm{y}$ anticoncepción, alcoholismo, tabaquismo y otras adicciones, eliminación de comportamientos sexistas, seguridad vial y ocio, primeros auxilios.

2. Actuación de asesoría; consiste en resolver dudas sobre alguno de los temas indicados de forma individual con el equipo "Forma Joven" o alguno de sus componentes (profesionales sanitarios) en el centro educativo.

3. Tablón "Forma Joven": ubicado en el centro educativo, junto al espacio para la asesoría. Se expondrán las actividades planificadas del Programa.

4. WEB "Forma Joven": en la página WEB del centro educativo, se creará un espacio donde se podrán consultar dudas sobre los temas del proyecto y/o de interés sanitario. El diseño de la página será diversificado y de respuesta inmediata.

\section{Discusión}

Las actividades realizadas por los profesionales del Centro de Salud (enfermera y trabajadora social) obtuvieron buena aceptación por parte del alumnado, del profesorado y de la Asociación de Padres y Madres de Alumnos. La participación en el taller fue numerosa (el alumnado estaba en horario académico) y las cuestiones planteadas aportaron luces sobre muchos "mitos" y datos de utilidad para mejorar en las actitudes de la población diana, en materia de educación sexual ${ }^{27,28}$. Todo ello se realizó en función de la valoración inicial con preguntas abiertas, las cuales mostraban por parte del alumnado un conocimiento deficiente en el tema y significativamente distinto cuando se analizaron las necesidades sexuales en función del género, al igual que en otros estudios ${ }^{5,29}$.

La escenificación es muy importante en las personas de estas edades. Cuando interpretan un papel pueden experimentar situaciones que no siempre conocen, permitiéndoles aprender de dicha representación. Todo esto se ve favorecido con el uso de dinámicas de grupo tales como juego de roles, "tormenta de ideas", etc ${ }^{30-32}$. Es por ello que en las sesiones de nuestro trabajo se escogieron algunas técnicas de escenificación de situaciones que suelen ocurrir en la vida real, con consecuencias no deseables y que se pueden evitar con coherencia y reflexión ${ }^{1-4,7}$.

Durante el desarrollo de la actividad, con el uso de dinámicas grupales se pretendía que los jóvenes eligiesen y tomasen una determinación o curso de acción a seguir en un momento de incertidumbre con consecuencias potencialmente nefastas, a partir de conocimientos básicos pero 
adecuados $^{1-5,7}$. Esto se debe en gran medida a que los conocimientos previos en materia de salud sexual entre el alumnado son escasos y están sesgados (como se indicó anteriormente), tanto por los recursos materiales disponibles ${ }^{30}$ como por la información adquirida, ya sea basada en la propia experiencia, o por los modelos a seguir ${ }^{31}$.

En consecuencia, la realización de dichas técnicas en nuestra actividad, contribuirían a disminuir el déficit de conocimientos, integrándolos de una manera práctica, y permitirían asentarlos de una forma más eficiente, así como ayudarían a los y las adolescentes en la toma de decisiones sobre los aspectos relacionados con la vida afectivosexual ${ }^{1,8,14}$. A pesar de las razones personales que pueden inducir a los jóvenes y las jóvenes a tomar decisiones en su vida sexual, es importante que conozcan el uso racional de los medios disponibles, para prevenir problemas perfectamente evitables ${ }^{32}$.

El soporte "on line" del proyecto ha sido presentado con el propósito de mantener una relación permanente con el alumnado. Este sistema de consulta a través de la red, tan usada por la juventud, favorece la promoción y prevención primaria de salud ${ }^{33}$.

La aplicación "on line” suscitó bastante expectación y curiosidad, lo cual era de esperar en la era de la tecnología en la que estamos inmersos. Este último aspecto del proyecto ha sido presentado para su implementación como una prestación más. Ésta se ofertará en un futuro, en la cartera de servicios de Atención Primaria de Salud. A través de ella los jóvenes y las jóvenes podrán realizar con total libertad cualquier tipo de consulta ${ }^{34}$. Este hecho repercutiría en una mayor difusión de conocimientos adecuados ${ }^{35} \mathrm{y}$ en una prevención de salud activa que evite situaciones no deseadas entre nuestros jóvenes ${ }^{36,37}$.

\section{Conclusiones}

A nuestro juicio, las estrategias sanitarias, en especial en relación con la sexualidad, deberían dirigirse a los y las adolescentes en un contexto diferente al del centro de salud y de acuerdo a sus necesidades; la actividad realizada manifiesta que la aplicación de éstas en el contexto educativo es una opción más que viable.

La información sobre sexualidad debe centrarse en combatir algunas creencias erróneas o mitos, muy arraigados, y focalizar estas actuaciones no sólo en mejorar conocimientos y actitudes, sino en generar cambios de conducta. La enfermera como agente de salud está capacitada para realizar actividades relacionadas con la promoción de una conducta sexual saludable.

Los conocimientos adquiridos en las distintas fases prácticas del proyecto han sido satisfactorios, tanto desde el punto de vista socio-sanitario como del educativo. Ello era de esperar tal y como según otros estudios realizados en el ámbito, con lo que se espera mejorar las conductas sexuales en esos estratos de edad.

Este estudio posibilita la implementación de un servicio telemático, el cual permitirá una mayor accesibilidad de los y las adolescentes a una información veraz y adecuada, independientemente de las normas sociales por las que se rigen y los motivos personales por los que declinan consultar con profesionales sanitarios. El impacto de este servicio podrá ser evaluado en los y las adolescentes. Además aportará nuevos datos sobre los que trabajar para abordar esta formación en la ESO.

Agradecimientos: Los autores agradecen la colaboración de todos los alumnos participantes así como de sus padres o tutores responsables por su conformidad en la participación de sus hijos, así como a los docentes implicados en este proyecto sin los cuales no habría sido posible su realización.

\section{Referencias}

1. Guerrero Masiá MD, Guerrero Masiá M, García-Jiménez E, Moreno López A. Conocimiento de los adolescentes sobre salud sexual en tres institutos de educación secundaria valencianos. Rev Ped Aten Primaria 2008; 10 (39): 49-58.

2. López F. La educación sexual. Madrid: Editorial Biblioteca Nueva; 2005.

3. Jussara Gue M. La educación sexual en tiempos del VIH/ SIDA. Enferm Global 2007; 6 (11): 1-18.

4. Kang $M$, Skinner $\mathrm{R}$, Usherwood T. Interventions for young people in Australia to reduce HIV and sexually transmissible infections: a systematic review. Sex Health 2010; 7 (2): 107-28.

5. De Irala J, Urdiain IG, López Del Burgo C. Analysis of content about sexuality and human reproduction in school textbooks in Spain. Public Health 2008; 122 (10): 1093-103.

6. Fernández Castillo R, Fenández Gallegos R. Educación 
para la salud, aprendizaje y cambio. Metas Enferm 2008; 11 (7): 58-61.

7. Wetmore CM, Manhart LE, Wasserheit JN. Randomized controlled trials of interventions to prevent sexually transmitted infections: learning from the past to plan for the future. Epidemiol Rev 2010; 32 (1): 12-36.

8. Macbeth A, Weerakoon P, Sitharthan G. Pilot study of Australian school-based sexual health education: parents' views. Sex Health 2009; 6 (4): 328-33.

9. Teva I, Bermúdez MP, Buela-Casal G. Characteristics of Sexual Behavior in Spanish Adolescents. Span J Psychol 2009; 12 (2): 471-84.

10. Vinet EV, Faúndez X, Larraguibel M. Adolescentes con trastorno por consumo de sustancias: Una caracterización de personalidad a través de las normas chilenas del MACI. Rev Med Chile 2009; 137 (4): 466-74.

11. Nikula M, Gissler M, Jormanainen V, Sevon T, Hemminki E. Sexual behaviour and lifestyles of young men in Finland, 1998-2005. Cross-sectional survey of military conscripts. Eur J Contracept Reprod Health 2009; 14 (1): 17-26.

12. Núñez Rocha GM, Alanís Alanís MJ, Alanís Salazar J, Salinas Martínez AM, Garza Elizondo ME, Villarreal Ríos E. Diferencias en la utilización de métodos de planificación familiar por mujeres adolescentes en Monterrey, México, según el modelo de educación sanitaria utilizado. Rev Esp Salud Pública 2005; 79 (1): 69-77.

13. González Hernando C, Sánchez-Crespo Bolaños JR, González Hernando A. Educación integral en sexualidad y anticoncepción para los/las jóvenes. Enferm Clín 2009; 19 (4): 221-4.

14. Arráez-Aybar LA, Núñez-Cortés JM, CarabantesAlarcón D, Lozano-Fernández R, Iglesias-Peinado I, Palacios-Alaiz E, et al. Adquisición de competencias transversales en alumnos de pregrado de Ciencias de la Salud en la Universidad Complutense: una experiencia positiva. Edu Méd 2008; 11 (3): 169-77.

15. Bermúdez MP, Castro A, Gude F, Buela-Casal, G. Relationship Power in the Couple and Sexual Double Standard as Predictors of the Risk of Sexually Transmitted Infections and HIV: Multicultural and Gender Differences. Curr HIV Res 2010; 8 (2): 172-8.

16. Wilson CM, Wright PF, Safrit JT, Rudy B. Epidemiology of HIV Infection and Risk in Adolescents and Youth. JAIDS 2010; 54 (Suppl. 1): S5-S6.

17. Rawson HA, Liamputtong P. Culture and sex education: the acquisition of sexual knowledge for a group of Vietnamese Australian young women. Ethn Health 2010; 15 (4): 343-64.

18. Lameiras Fernández M, Carrera Fernández MV, Núñez Mangana AM. Evaluación de un programa de educa- ción sexual con adolescentes: una perspectiva cualitativa. Diversitas 2006; 2 (2): 193-204.

19. Rodríguez Castilla F, Ramos Morcillo AJ, Fernández Salazar S. Infecciones de transmisión sexual. Metas Enferm 2009; 12 (5): 50-5.

20. Gómez Galán MP, Gómez-Galán R, De la Peña Tejeiro E, López Gómez MJ, Garrido González J, Herrero Olea A. Calidad de las clases de educación sexual en la enseñanza secundaria. Metas Enferm 2003; 6 (55): 59-64.

21. Paulin Baraldi AN, Prado Daud Z, De Almeida AM, Azevedo Gomes F, Spanó Nakano AM. Embarazo en la adolescencia: Estudio comparativo de las usuarias de maternidades públicas y privadas. Rev. Latino-Am. Enfermagem 2007; 15 (número especial): 1-7.

22. Wyatt Seal D, Bogart LM, Ehrhardt AA. Small Group Dynamics: The Utility of Focus Group Discussions as a Research Method. Group Dyn-Theor Res 1998; 2 (4): 253-66.

23. Pérez Milena AP, Olmedilla MR, Gallardo IM, Jiménez Pulido I, Martínez Fernández ML, Pérez Milena R. Motivations for alcohol use among adolescents in an urban high school. Aten Primaria 2010; 42 (12): 604-11.

24. Ingram J, Salmon D. Young people's use and views of a school-based sexual health drop-in service in areas of high deprivation. Health Educ J 2010; 69 (3): 227-35.

25. Ley Orgánica del 2/2006 de 3 de Mayo. Publicada en el Bol Ofic Estado no 106 4/5 2006. Madrid; 2006.

26. Hsu HY, Lien YF, Lou JH, Chen SH, Wang RH. Exploring the effect of sexual empowerment on sexual decision making in female adolescents. J Nurs Res 2010; 18 (1): 44-52.

27. Estepa Osuna MJ, Granados Matute AE, Barroso Vázquez M. Importancia del entrenamiento para la entrevista clínica de valoración inicial: aspectos relevantes. Enferm Global 2008; 7 (14): 1-14.

28. Jordán Jinez ML, Molina de Souza JR, Pillon SC. Uso de drogas y factores de riesgo entre estudiantes de enseñanza media. Rev. Latino-Am. Enfermagem 2009; 17 (2): 246-52.

29. Frappier JY, Kaufman M, Baltzer F, Elliott A, Lane M, Pinzon J, et al. Sex and sexual health: A survey of Canadian youth and mothers. Paediatr Child Health 2008; 13 (1): 25-30

30. Hoppe MJ, Graham L, Wilsdon A, Wells EA, Nahom D, Morrison DM. Teens speak out about HIV/AIDS: focus group discussions about risk and decision-making. J Adolesc Health 2004; 35 (4): 27-35.

31. Vilela Borges AL, Nakamura E. Normas sociales de iniciación sexual entre adolescentes y relaciones de género. Rev. Latino-Am. Enfermagem 2009; 17 (1): 94-100.

32. Sánchez Díaz E, Villar Luis MA, Rasso GH, Pillon. 
Educar adolescentes en conductas sexuales saludables - M. A. García et al

Análisis prospectivo de la enseñanza de prevención de consumo de alcohol y salud reproductiva en facultades/ escuelas de educación de Lima-Perú. 2003-2004. Rev. Latino-Am. Enfermagem 2005; 13 (número especial): 854-62.

33. Aslan D, Sahin A. Adolescent peers and anti-smoking activities. Promot Educ 2007; 14 (1): 36-40.

34. Saura Sanjaume S, Fernández de Sanmamed Santos MJ, Vicens Vidal L, Puigvert Viu N, Mascort Nogué C, García Martínez J. Percepción del riesgo de contraer una enfermedad de transmisión sexual en población joven. Aten Primaria 2010; 42 (3): 143-8.
35. Carrera-Fernández MV, Lameiras-Fernández M, Foltz ML, Núñez-Mangana AN, Rodríguez-Castro Y. Evaluación de un programa de educación sexual con estudiantes de Educación Secundaria Obligatoria. IJCHP 2007; 7 (3): 739-51.

36. Dias VP, Witt RR, Silveira DT, Kolling JH, Fontanive P, De Castro Filho ED, et al. Telenursing in primary health care: report of experience in southern Brazil. Stud Health Technol Inform 2009; 146: 202-6.

37. Navarro M, Mateo MJ. Informe juventud en España. Madrid: Ed. Servicio de publicaciones del Instituto de la juventud; 1993. 